\title{
A SYSTEM FOR NATURAL LANGUAGE \\ UNMARKED CLAUSAL \\ TRANSFORMATIONS IN TEXT-TO-TEXT
}

\section{APPLICATIONS}

\author{
A Thesis \\ presented to \\ the Faculty of California Polytechnic State University, \\ San Luis Obispo
}

In Partial Fulfillment of the Requirements for the Degree Master of Science in Computer Science

by

Daniel Steven Miller

June 2009 
(C) 2009

Daniel Steven Miller

ALL RIGHTS RESERVED 


\section{COMMITTEE MEMBERSHIP}

TITLE:

AUTHOR:

DATE SUBMITTED:

COMMIT'TEE CHAIR:

COMMIT'TEE MEMBER:

COMMITTEE MEMBER:
A System for Natural Language Unmarked Clausal

Transformations in Text-to-text Applications

Daniel Steven Miller

June 2009

Franz J. Kurfess

Gene Fisher

Christopher Clark 


\begin{abstract}
A System for Natural Language Unmarked Clausal Transformations in Text-to-text Applications

Daniel Steven Miller
\end{abstract}

A system is proposed which separates clauses from complex sentences into simpler standalone sentences. This is useful as an initial step on raw text, where the resulting processed text may be fed into text-to-text applications such as Automatic Summarization, Question Answering, and Machine Translation, where complex sentences are difficult to process. Grammatical natural language transformations provide a possible method to simplify complex sentences to enhance the results of text-to-text applications. Using shallow parsing, this system improves the performance of existing systems to identify and separate marked and unmarked embedded clauses in complex sentence structure resulting in syntactically simplified source for further processing. 


\section{ACKNOWLEDGMENTS}

Special thanks to my advisor Dr. Franz Kurfess, for being continually available and supportive, and the members of my committee, Dr. Gene Fisher and Dr. Christopher Clark for helping me clarify and focus this thesis. I would like to thank my parents for helping to get me

through and inspire me to do my best. Thanks especially to my wife Sarah, and my children Lucas and Lydia for spurring me on to bring this to completion. 


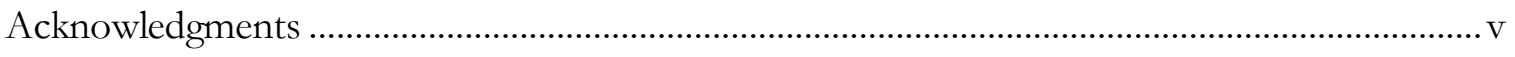

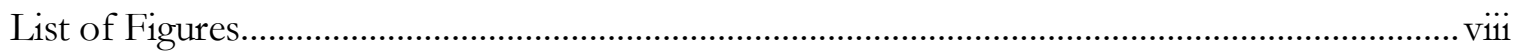

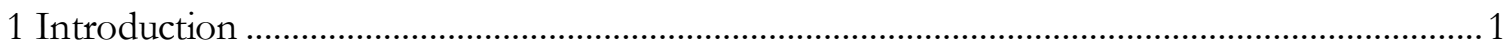

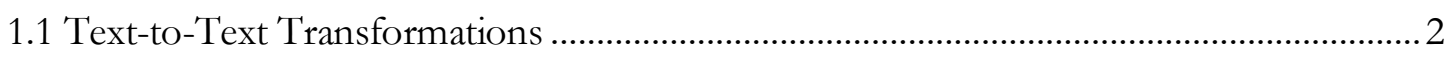

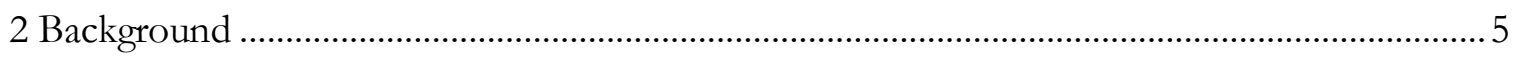

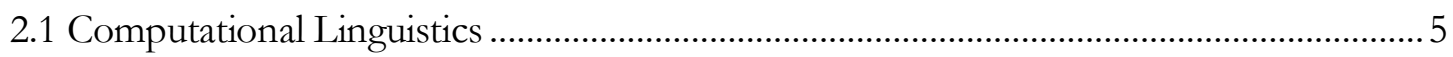

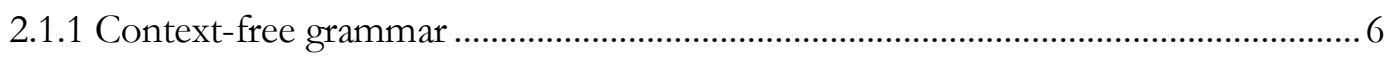

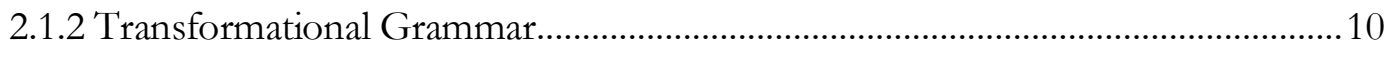

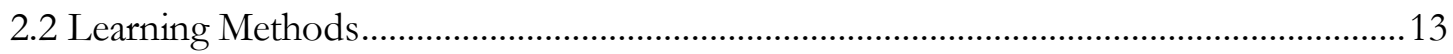

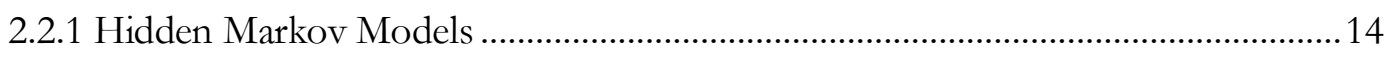

2.2.2 Transformation-based Learning ........................................................................ 15

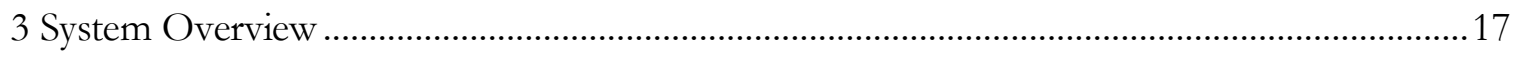

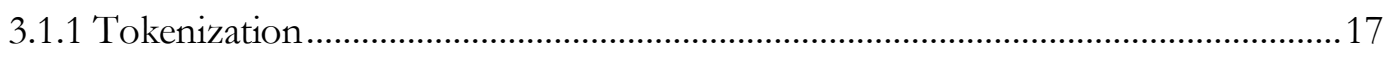

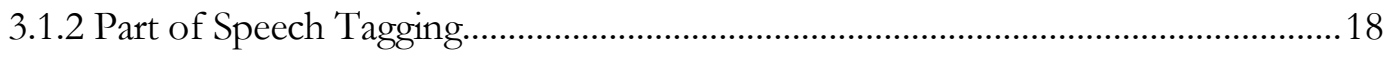

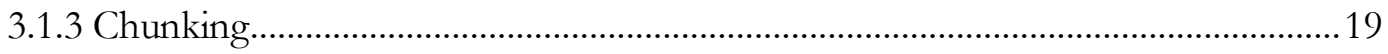

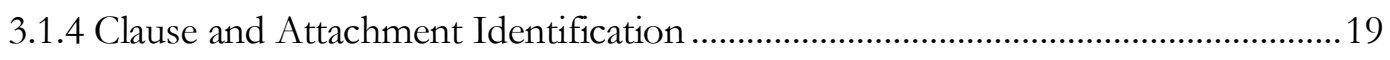

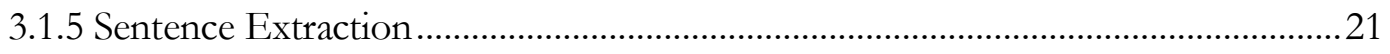

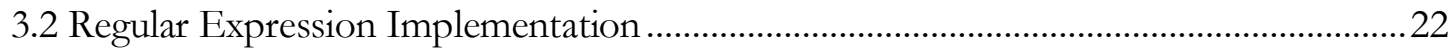

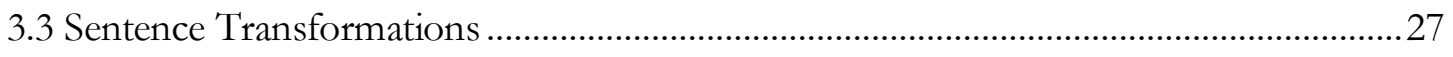

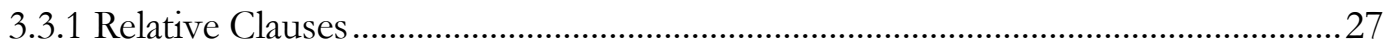

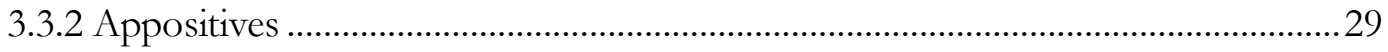




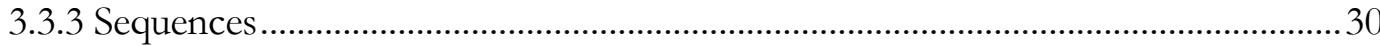

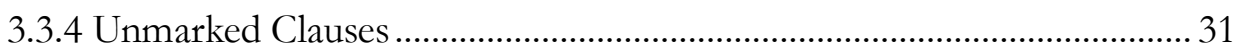

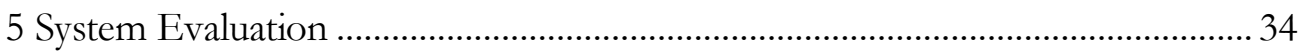

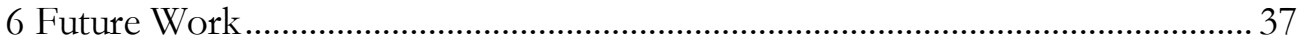

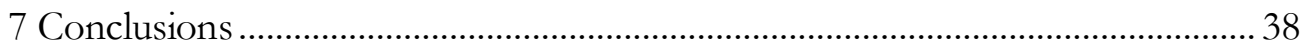

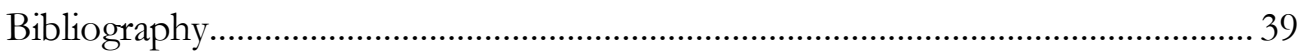

Appendix 1- Example Output.......................................................................... 42 


\section{LIST OF FIGURES}

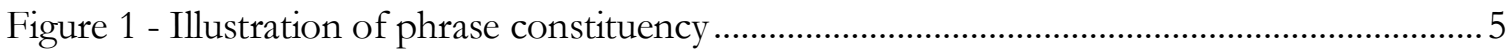

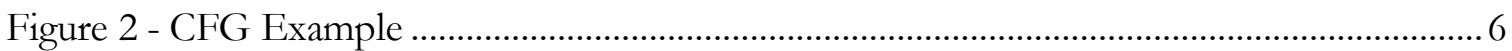

Figure 3 - Generation of "The man took the book".................................................................

Figure 4 - Parse Tree Example "The man took the book." ........................................................... 8

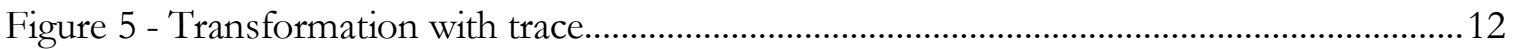

Figure 6 - System Pipeline.................................................................................................... 17

Figure 7 - Regular expression relative clause identification algorithm ........................................20

Figure 8 - Siddharthan relative clause identification algorithm.................................................21

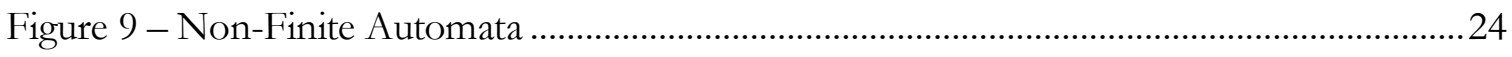

Figure 10 - Relative Clause Identification Comparison ................................................................ 34

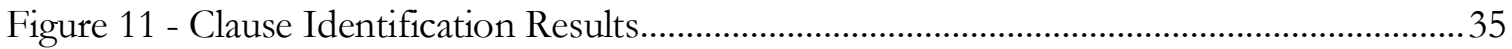




\section{INTRODUCTION}

With the expansion of massive amounts of digitized text available, most prominently through the World Wide Web, advanced information retrieval (IR) techniques are more necessary than ever. While language information is currently treated at the document or sentence level (Mani and Maybury 1999), this is not the expected or optimal solution, often requiring people to search through the clumsy, out of context results to find the information they require. For instance, in the Question Answering track of the Text REtrieval Conference (TREC) 2000 had the question, What is the capital of Kosovo? as one of its reported examples (Voorhees 1999). Answers ranged from 0 miles northwest of Pristina, five demonstrators which was judged correct to protesters called for military intervention to end "the Albanian uprising." $</ P><P>$ At Vucitrn, 20 miles northwest of Pristina, five demonstrators were reported injured, apparently in clashes with police. $\quad\langle/ P><P>$ Violent clashes were also repo which was judged incorrect. The second was judged incorrect even with the correct answer within the text because of the ambiguity of two possible city names in the snippet. A much better solution would be to have a short, direct sentence with the name of the city.

Systems that answer queries for text retrieval must accept complex sentences with complex references to other parts of the text within the search data. These data often contain linguistic barriers that current systems are unable to handle which will often obscure relevant information (Chandrasekar, Doran and Srinivas 1996). Keyword-based techniques have improved IR at the document level, but have done little to extract the necessary data within the document at the sentence or sub-sentence level. This thesis presents an architecture for a 
preprocessing method which augments IR systems to enhance their results by identifying and transforming several types of clauses within the text.

The goal of this thesis is to use shallow parsing and regular expressions for acquiring and transforming clausal information from sentences, resulting in simplified sentences with a similar degree of accuracy as Siddharthan (Siddharthan 2003), but without resorting to a handcoded solution. Furthermore, Siddharthan's algorithm is improved upon so that clausal boundaries are found using grammatical parts of speech instead of punctuation and word cues. In this thesis, a system is presented for text processing methods to move from rule-based to a potentially machine learning based approach.

\subsection{TEX T-TO-TEXT TR A N F O R M A T I N S}

Many of the most popular natural language applications today are text-to-text applications, that is, applications which both accept and produce text as opposed to applications which perform data analysis or parsing over text. These include machine translation, summarization, and question answering. In each case, the primary focus of each is separate from modifying the base text itself. In machine translation, the focus is in translating the words and phrases from the input to the target language. In summarization and question answering, focus is on the ability to accurately find the most salient sentences pertaining to the query (Erkan 2004). The TREC 2003 and 2004 question answering track shows an increasing emphasis on linguistic 
parsing (Voorhees 2004, Voorhees 2005). Challenges in the TREC QA track first began in 1999 with document retrieval, then passage retrieval within the documents, and finally in 2002, the exact answer to factoid questions such as Who invented the paper clip? Introduced in 2003 were definition questions (Voorhees 2004). These questions are broad, open-ended questions such as, What is mold? requiring retrieval across multiple documents. The TREC 2004 QA track was designed to incorporate even more definition questions through a tell me more style question at the end of each question series (Voorhees 2005). Using deeper linguistic constructs has proven useful (Jinxi Xu 2004) to definition question answering, earning the highest score in the TREC 2003 QA track definition question series.

While deeper linguistic constructs are useful, relatively few efforts have been made to separate the task of initial parsing and final text-to-text processing from the main task of salience (relevance) scoring and translation. Instead, text-to-text processing is handled as a part of the main task, unique to each implementation. One effort to split this task is the application of surface realization-generating the final text from a discourse plan-in natural language generation (NLG) to text-to-text tasks like summarization and machine translation. In NLG, the output of an expert system or other non-language source is finally "realized" or expressed as a human readable sentence at the stage of surface realization. Since many surface realization implementations require deep subject-verb or verb-object relations unavailable to NLP applications, only the recent formalization of "interleave disjunction lock" or IDL-expressions 
for natural language parsing (Mark-Jan Nederhof 2004) has allowed its application to text-totext applications to be considered (Marcu 2005).

The most basic text-to-text transformations that process deeper than the sentence level are sentence simplification techniques. Text simplification by itself is useful for generating closedcaptioned text and other limited channel devices, aiding adult English learners, and aiding people with language disabilities like aphasia (Carroll, et al. 1999). 


\section{BACKGROUND}

\subsection{O M P U T A T I O N A L I N G U I S T I C S}

Parsing Natural Language first began before computers with linguistics through formalizing grammar in language so that a delineation could be made between grammatical and ungrammatical sentences (Ouhalla 1999). Sentences contain groups of words that behave as a single unit or phrase, called a constituent. A constituent may be a noun phrase such as Kermit the Frog, an adjective phrase such as extremely clever, a prepositional phrase such as down by the river, a verb phrase such as killed the rabbit and a handful of others. Evidence of the validity of constituency is the phrase's ability to be placed in different locations throughout the sentence, as in Figure 1.

On May twenty-fifth I'd like to fly from California to New York. I'd like to fly on May twenty-fifth from California to New York. I'd like to fly from California to New York on May twenty-fifth.

* On May I'd like to fly twenty-fifth from California to New York. * On I'd like to fly May twenty-fifth from California to New York.

Figure 1 - Illustration of phrase constituency 


\subsubsection{CONTEXT-FREE GRAMMAR}

The most common way of modeling a constituency is a Context-free grammar (hereafter CFG), which is also called a Phrase Structure Grammar or Backus-Naur Form. These models were formalized by Chomsky in 1956 (Chomsky 1956), and independently by Backus in 1959 . A CFG may be represented by $G=<T, N, S, R>$ in which $S$ is a nonterminal start symbol, $\mathrm{T}$ is the set of terminals, or lexicon, and $\mathrm{N}$ is the set of nonterminals. Structuring the CFG is a set of rules $\mathrm{R}$, or productions, in the form of $\mathrm{X} \rightarrow \gamma$ where $\mathrm{X}$ is a nonterminal and $\gamma$ is a sequence of terminals and nonterminals applied to $\mathrm{N}$ and $\mathrm{T}$ to generate some language $\mathrm{L}$.

The example in Figure 2 defines a formal language from (Chomsky 1956). A formal language differs from a natural language in that a formal language is defined solely by the rules in the

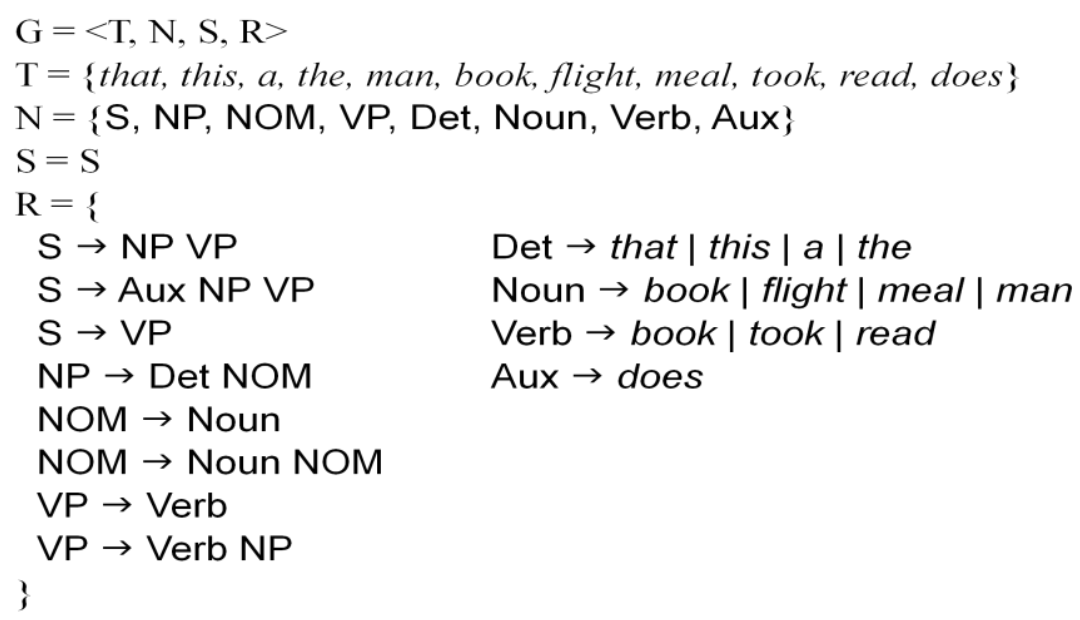

Figure 2 - CFG Example 
CFG. The application of the rules generates all possible grammatical sentence for our formal language $G$. Any sentence formed that cannot be derived from $G$ are called ungrammatical for this particular formal language. In the example, NP stands for noun phrase, VP for verb phrase, NOM for nominal. A nominal can be one or more nouns. In linguistics, this is called a generative grammar, since the language is defined by the set of possible sentences "generated" by the grammar. We may generate the sentence The man took the book. as in Figure 3.

The generation of a sentence using a CFG is called derivation. In order to represent the complete derivation, a parse tree is commonly used as in Figure 4. The parse tree begins at the top with the nonterminal start symbol S. NP and VP are then derived from S using the CFG rules in the next level down. The parse tree ends at the bottom with the words which make up the sentence.

$S \rightarrow N P V P$

$\rightarrow$ Det NOM VP

$\rightarrow$ The NOM VP

$\rightarrow$ The Noun VP

$\rightarrow$ The man VP

$\rightarrow$ The man VP NP

$\rightarrow$ The man Verb NP

$\rightarrow$ The man took Det NOM

$\rightarrow$ The man took the NOM

$\rightarrow$ The man took the Noun

$\rightarrow$ The man took the book

Figure 3 - Generation of "The man took the book" 


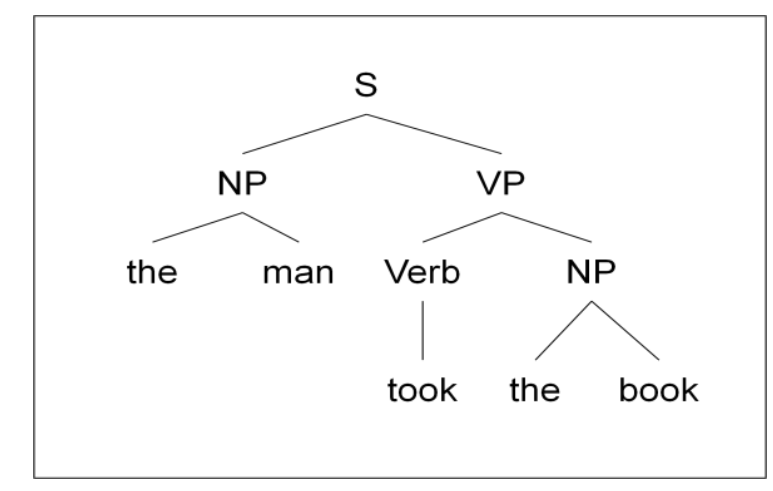

Figure 4 - Parse Tree Example "The man took the book."

While deriving sentences from a CFG is straight forward, finding the correct derivation of a sentence is much harder. This task is called parsing, where given a sentence, one must find the correct derivation. It must be noted that a particular sentence may have dozens or more parse trees which all satisfy the rules of a given grammar. Parsing algorithms have been handled computationally as search algorithms, where two popular algorithms are defined by their starting points. Top down parsing begins at the start symbol S, and generates a derivation using the grammar rules given. Bottom up parsing begins with the terminal words and using the grammar rules in reverse to generate the derivation. Parsing itself is computationally expensive. The popular Earley (Earley 1968) algorithm implements a parallel top-down approach which gives a worst case behavior of $\mathrm{O}\left(\mathrm{N}^{3}\right)$, where $\mathrm{N}$ is the number of words in the input. Parsing the complete derivation of the sentence, however, is not necessary for many tasks in natural language processing. A common method used is called shallow parsing, where the individual constituents of a sentence are identified, but their derivations are left unknown. 
Shallow parsing itself may be split into several tasks itself. The first is called tokenization, where a string is split into sentences, words, and punctuation. Tokenization is a simple, but not trivial task, and errors at this stage prove disastrous to the remaining tasks. The second task is called tagging, where each token is identified by its part of speech, which may be a noun a possessive ending, a comma, or a third-person singular present verb. Tag sets themselves may differ greatly, identifying larger or smaller differences in parts of speech. Two popular tag sets are noted by the corpuses in which they are used, the Brown tag set (Francis and Kucera 1982), and the Penn Treebank tag set (Santorini 1991). The third task in shallow parsing is called chunking. Chunking results differ from the full parse of a sentence because chunks are defined as non-overlapping consecutive groups. In a full parse, one chunk may be the constituent of another, but in shallow parsing such information is not retrieved. A shallow parser may run each of these tasks in succession, or generate the tokenized, tagged, and chunked data in one pass. A shallow parse of the example sentence would appear: [NP The/DT man/NN] [VP took/VBD] [NP the/DT book/NN]. Tags are shown after the slash '/' for each word, while chunks are divided by square brackets '[' with their constituency preceding the text.

CFG is not the only or most recent or accepted method of representing language or syntax structure. In linguistics, there are multiple competing philosophies of language (Martin 2000), none of which have won out or completely described natural language without exceptions. While constituency gained popularity early on in the United States, European syntactitians 
retained the earlier word-based dependency grammars, which determine syntax by individual word relations. Furthermore, CFG itself has been modified into a set of more abstract contextfree templates known as X-bar schemata (Ouhalla 1999), which is still in active development. While a large number of computational models were based on context free grammars due to early work in computational parsing these grammars, dependency grammars have gained popularity with statistical parsers.

\subsubsection{TRANSFORMATIONAL GRAMMAR}

Moving beyond phrase structure are the more complex grammatical transformations. Transformational Grammar (Ouhalla 1999) is a version of Generative Grammar that developed when linguistics moved from the mechanics of language to the underlying mental processes of a broader range of the properties of language. Transformational Grammar introduced a set of construction-specific transformations on different types of sentences which would either leave the meaning of the sentence the same, or accomplish a specific desired grammatical change.

In order to understand transformations, we must first have a deeper understanding of sentence structure. It is tempting to consider some linear relationship between words in a sentence. In English, we come across many sentences with a subject-verb-object linear structure, but this is merely a by-product of the geometrical aspects of the structures generated by Phrase Structure 
rules. Considering the tree structure of language it is possible to then observe specific movements of structures as more than the NP movement introduced earlier. In Figure 5, we see two tree structures of the sentences I can solve this problem. And This problem, I can solve. The transformation in effect is called the Lexical Insertion Rule, where item $\mathrm{X}$ is inserted under terminal node $\mathrm{Y}$ where $\mathrm{Y}$ corresponds to the categorical features of $\mathrm{X}$, and YP corresponds to the subcategorization properties of $\mathrm{X}$. The second tree structure has a $\mathrm{t}(\mathrm{race})$ symbol in the object position of the verb. Trace is a unique category in sentence structure in that it has no lexically realized form, but instead marks grammatically the result of a transformation that has taken place. 


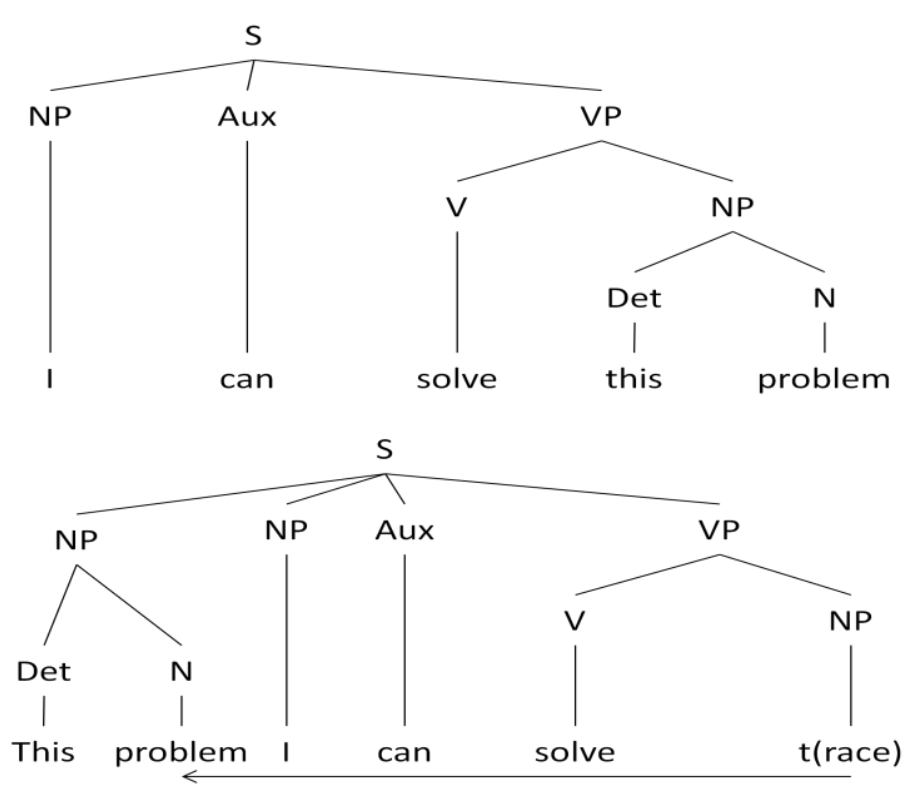

Figure 5 - Transformation with trace

Trace does not require a purposeful linguistic transformation to exist. It exists implicitly as part of the grammatical structure of a sentence. Consider the colloquial "wanna" as a contraction of the words "want" and "to" when they are adjacent.

1. I want to read this novel.

2. I wanna read this novel.

Now if there is a transformation that takes place, we can show the existence of a trace in the resulting sentence:

1. This novel, I want to be considered for a prize. 
2. *This novel, I wanna be considered for a prize.

3. [NP This novel $]_{i}$, I want $[N P t]_{i}$ to be considered for a prize.

Since the trace comes between "want" and "to", they cannot contract to "wanna", showing the grammatical visibility of the trace symbol.

\subsection{E A R N I G M E TH O D S}

NLP was exclusively rule-based for some time, but then statistical methods such as Hidden Markov Models have been one of the most favored techniques for computational linguistics (Martin 2000). Statistical techniques have the advantage of being relatively hands-off while producing generally positive results. There are problems with statistical techniques though. First, the resulting output of a training period is a set of interdependent statistical weights which do not provide any insight into the methods used by the trained system. The programmer may then blindly or intuitively adjust certain aspects of the training, but the programmer is essentially feeling around in the dark.

A compromise approach between rule-based and statistics based methods is Transformation Based Learning (Brill, Transformation-based error-driven learning and natural language processing: A case study in part of speech tagging 1995), which generates a set of rules as the 
result of its training. These rules can be and have been manipulated by hand after training has occurred in order to improve performance of the final algorithm.

\subsubsection{HIDDEN MARKOV MODELS}

Hidden Markov Models are one of the most important machine learning models in natural language processing. To introduce Hidden Markov Models, one must first be introduced to Markov Chains (Martin 2000). An extension of finite state automata, the Markov Chain is a weighted special case automaton where the input sequence determines a unique set of states. A Markov Chain is made up of a set of states $Q=q_{1} q_{2} \ldots q_{N}$, a set of transition probabilities $A=a_{01} a_{02} \ldots a_{n 1} \ldots a_{n n}$ and start and end states. Since each transition $a_{i j}$ has the probability $p\left(q_{j} \mid q_{i}\right)$ the sum of the outgoing transitions is always equal to 1 . Start states may be represented as a

distribution over all states $\pi$ where $\pi_{\mathrm{i}}$ expresses the probability $p\left(q_{i} \mid S T A R T\right)$. In a first order Markov chain, the probability of a particular state $q_{i}$ is dependent only on the previous state, so $p\left(q_{i} \mid \ldots q_{i-1}=p\left(q_{i} \mid q_{i-1}\right)\right.$. From a Markov chain, we can compute the probabilities of a set of states using the transition and start state probabilities.

While Markov chains are useful for observable events, many events in NLP are not observable. In part-of-speech tagging, for instance, the observed events are words rather than tags. The correct tags have to be inferred from the word sequence given. Hidden Markov Models (HMM) allows for the modeling of hidden events based upon observed events. As 
with a Markov Chain an HMM is made up of of a set of states $Q=q_{1} q_{2} \ldots q_{N}$, a set of transition probabilities $A=a_{01} a_{02} \ldots a_{n 1} \ldots a_{n n}$, and an initial distribution over states $\pi$, but an HMM also has a set of observations $O=0_{1} o_{2} \ldots o_{N}$ with each observation drawn from a vocabulary $V=v_{1} v_{2} \ldots v_{V}$ and a set of observation likelihoods $B=b_{i}\left(0_{0}\right)$. A first-order HMM not only requires that the probability of a state is only dependent on the previous state, but also the probability of an output observation is dependent only on the state producing the observation.

\subsubsection{TRANSFORMATION-BASED LEARNING}

Transformation-based learning (Brill, Transformation-based error-driven learning and natural language processing: A case study in part of speech tagging 1995, Brill, A simple rule-based part-of-speech tagger 1992) (hereafter TBL) is the only remaining rule-based algorithm in Natural Language Processing that has the advantages of statistical methods, but instead of blocks of statistical data, a relatively simple rule-set is produced. Several advantages the rule-set provides are that errors can be pinpointed to certain rules in the rule-set, and the rule-set is intelligible enough to be hand-tweaked for further improvements.

TBL is an error-driven machine learning technique that works by first assigning an initial classification to the data, and then searches through a set of possible transformations, selecting the transformation which most decreases the error in the training set. This process repeats until no transformation will decrease the error rate in the training set. Each transformation, or 
rule, consists of a predicate and a target. In the case of part of speech tagging, two rule-sets are applied to the data after its initial classification. The first are a set of lexical rules, which incorporates the letters that make up the word being tagged, including prefixes, suffixes and capitalization. The second is a set of contextual rules, which incorporate the surrounding words and tags in order to assign a correct part of speech tag.

TBL has several attractive qualities beyond the generated rule-set. Since TBL is directly errordriven, it is resistant to over-training. It can automatically integrate heterogeneous types of knowledge without explicit modeling. Furthermore it outperforms Hidden Markov Model techniques (Ramshaw and Marcus, Exploring the statistical derivation of transformational rule sequences for part-of-speech tagging 1994) that had taken over similar NLP tasks before its arrival.

TBL was first proposed by Eric Brill (Brill 1992, Brill 1995), and subsequently modified to both improve the training speed of the algorithm (Florian and Ngai 2001, Ramshaw and Marcus, Exploring the statistical derivation of transformational rule sequences for part-ofspeech tagging 1994) and expanding the scope of the original algorithm beyond part-of-speech tagging to chunking (Ramshaw and Marcus 1995), parsing (Brill 1996), and handwritten character segmentation (Kavallieratou, et al. 2000). 


\section{SYSTEM OVERVIEW}

The overall structure of the system is separated into five parts: tokenization, part-of-speech tagging, chunk tagging, clause and attachment identification, and sentence extraction. The system was programmed in Python using the natural language toolkit (NLTK) (Bird 2002). The novel parts of this system are in the clause identification and sentence transformation methods.

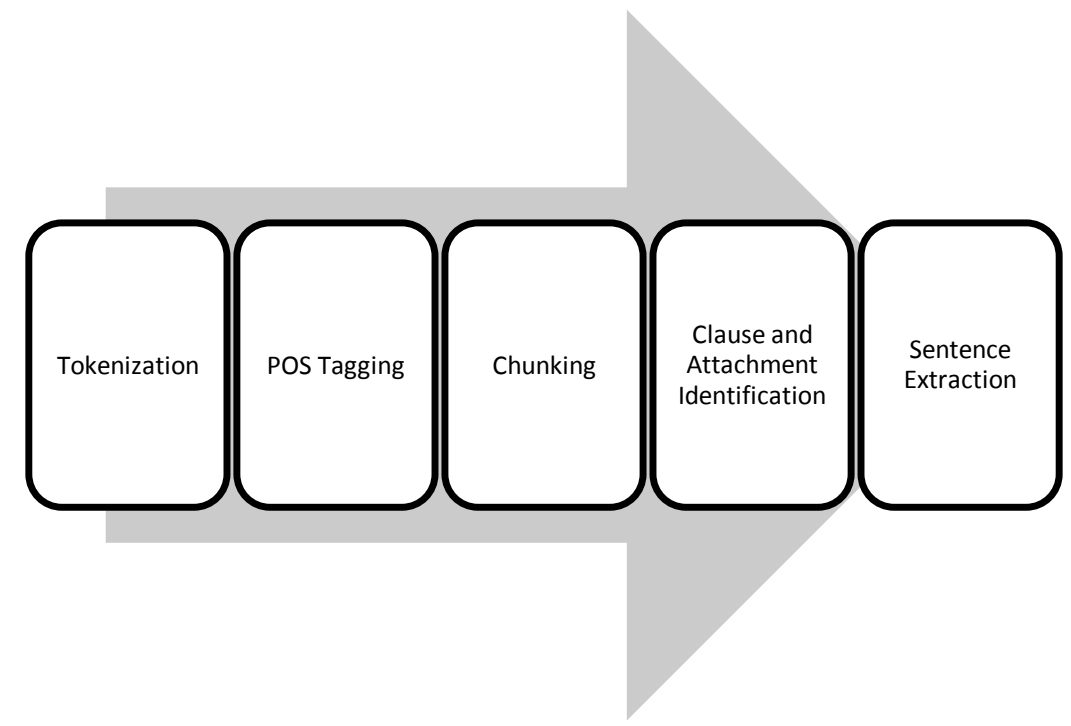

Figure 6 - System Pipeline

\subsubsection{TOKENIZATION}

In tokenization, a string of characters is grouped into tokens. Normally this is an array of words and punctuation in a sentence. So the string, Mary's doll had red hair. gets tokenized into, 
["Mary", "'s", "doll”, "had", "red", "hair", "."]. This facilitates further processing by allowing the next steps to concentrate on a token rather than a stream of characters. The tokenization step in this system is from the Montytagger (Liu 2003), which is a transformation based part of speech tagger written in Python. For the purpose of this system the numeric identifier in the Montytagger tokenizer was expanded to handle numbers larger than 1 million and currency with more than two digits after the decimal point.

\subsubsection{PART OF SPEECH TAGGING}

Part of speech tagging takes the tokens produced at the tokenization step and applies a part of speech tag to each token. In this step, the tags are applied using transformation-based learning. The tokens, ["Mary", "'s", "doll”, "had", "red", "hair", “."], is input into the part of speech tagging stage and results with the output, ["Mary"/NNP, "'s"/POS, "doll"/NN, "had"/VBD, "red"/JJ, "hair"/NN, “."/.]. There are several problems with training taggers from scratch. The first is that the process can take weeks of computing time. The second is that the freely available tagged corpuses are much smaller than those for which rulesets are available. The fnTBL system has both part of speech and chunk tagging rulesets available which used the large and well-established Penn Treebank Wall Street Journal corpus as its source. The system interprets fnTBL's trained rulesets and applies them to the tokens. 


\subsubsection{CHUNKING}

In the chunking stage, the tagged tokens are separated into non-overlapping phrases called "chunks". The chunks are groups of adjoining tokens which form phrases that constitute the most basic level of constituents within a sentence. In this implementation, chunks are separated using chunk tags at each token. At this stage, the input, ["Mary"/NNP, "'s"/POS, "doll"/NN, "had"/VBD, "red"/JJ, "hair"/NN, "."/.], would be further tagged, ["Mary"/NNP/BNP, “'s"/POS/B-NP, "doll"/NN/I-NP, "had"/VBD/B-VP, "red"/JJ/B-NP, "hair"/NN/I-NP,

"."/./O]. In order to form groups, each chunk tag is prefixed with B- to mark the beginning of the chunk or I- to continue the previous chunk. A second trained set of rules from fnTBL were used to apply chunk tags to the tokens.

\subsubsection{CLAUSE AND ATTACHMENT IDENTIFICATION}

At the clause and attachment identification stage, the tagged and chunked tokens are used to identify the attachment and clause beginnings and endings. The method used in this is a custom regular expression engine which uses a tagged token as a unit rather than a character. The clause identification algorithm for appositives and relative clauses are based on Siddharthan's (Siddharthan 2003) work in rule based clause identification. The difference can 
be seen in Figure 8 and Figure 7, where the pseudocode from Siddharthan has been translated into a regular expression.

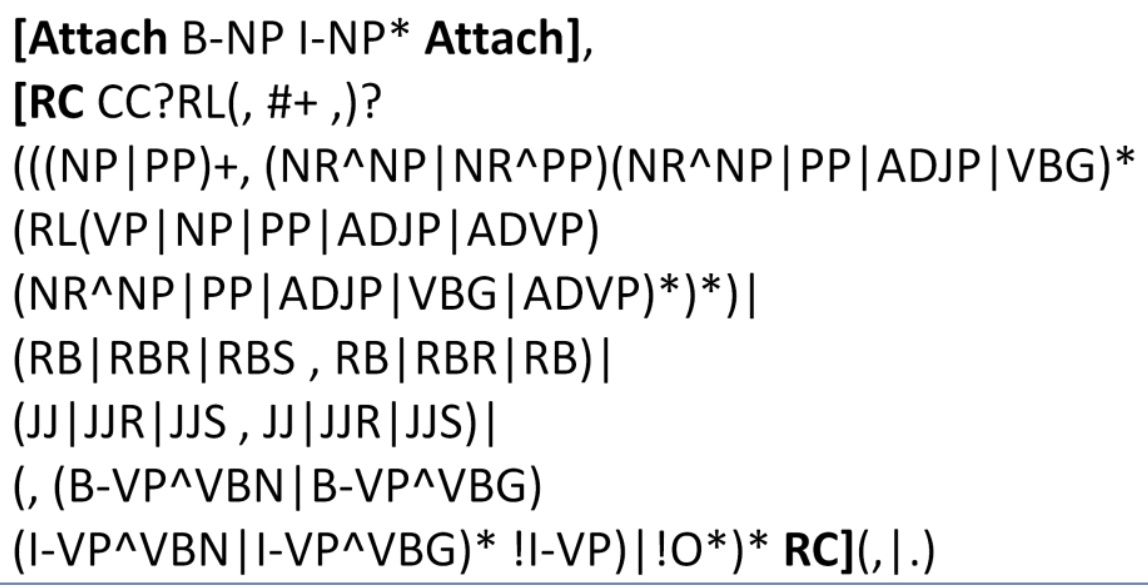

Figure 7 - Regular expression relative clause identification algorithm 


\section{Decide-Non-Restrictive-RC-Boundaries}

1. LET $n$ be the number of commas between ", $\{$ who $\mid$ which $\}$ " and the end of the sentence $(</$ S1 $>)$ or enclosing clause $(</$ SIMP $-\ldots>)$.

2. IF $n=0$ THEN clause extends till the end of sentence

3. IF $n>0$ THEN a decision needs to be made at each comma as follows:

4. IF the relative pronoun is immediately followed by a comma THEN Jump to the token after the next comma

5. FOR each comma (scanning from left to right) DO

(a) IF followed by an appositive (appositive determination is described in section 3.4.2) THEN INTERNAL comma

(b) IF followed by a verb group THEN

i. IF the verb has POS "VB $\{\mathrm{N} \mid \mathrm{G}\}$ " THEN INTERNAL comma

(c) IF an implicit conjunction of adjectives or adverbs like "JJ, JJ" or "RB, RB" THEN INTERNAL clause

(d) IF it is a Pronoun $X$ clause where Pronoun $X=\{$ who $\mid$ which $\}$ THEN

i. IF ", CC Pronoun_X" THEN INTERNAL clause and DELETE "Pronoun_X"

ii. IF ", \{who|which|that $\}$ " THEN INTERNAL comma

6. ELSE by default end clause on first comma

Figure 8 - Siddharthan relative clause identification algorithm

\subsubsection{SENTENCE EXTRACTION}

Sentence extraction takes the marked clauses and attachments and performs several operations on them, extracting stand-alone sentences. The clause may be removed as in the case with appositives, or joined with its attachment to form a separate sentence. For relative clauses, the relative pronoun must also be identified and dropped from the extracted sentence. For sequences, each clause may stand on its own within the sentence. This was done through a simple hand-coded algorithm, which would print out each extracted sentence. 


\subsection{R E G U A R EX PRES I O N I M P E M E N T A T I O N}

In order to identify the clauses for extraction several approaches were considered, including a variety of machine learning techniques. Since clause identification concerns more complex relationships between words, more advanced machine learning techniques and even handcoded algorithms become relevant to the problem. One main obstacle to a machine-learning technique is the lack of training corpa available. The CoNLL 2001 shared task (Dejean and Sang 2001) may have provided a training corpus had it identified clause types rather than simple boundaries. Since only a handful of clause types lend themselves to extraction, and different techniques are required for extraction on differing clausal categories, clausal type information is essential to sentence extraction.

The technique used for clause identification is a similar approach to Thompson's regular expression algorithm (Thompson 1968). Thompson's algorithm generates a non-deterministic finite automata (NFA) from a postfix regular expression input to determine matches on a string. In this system, the expression must match across an array of tokenized and tagged units rather than an array of characters. This introduces several challenges in generating the capability to express a clausal identification algorithm within an input expression which will be further discussed later.

Thompson's algorithm uses a stack-based approach to generate the NFA, which pulls tokens off a postfix-style regular expression and generates the NFA iterively from each subexpression. 
This system uses a translator to create a postfix-style expression from an infix-style expression input. The resulting expression is used to generate the NFA using a similar approach to Thompson. The main difference with this algorithm is the data which the expression must match against. Instead of a simple character array, each token has three components of which one or more may need to be matched against the expression. Secondly, instead of a simple character match, the match may only be on the whole word, the part of speech tag, or the chunk tag. There are seven operators that this engine handles in the NFA structure and three in the matching code. The seven operators include concatenation, or-style alternation, matching of zero or more, matching of one or more, matching of zero or one instance, simple tag concatenation, and tag continuation. The three handled in the matching code are matching across a single token, negation, and wildcard matches.

In each of the seven operators in Figure 9, the generator needs to create a NFA, and connect them until a terminal matching state is reached. For states such as concatenation, simple tag concatenation, and continuation, the NFA simply connects one input arrow to one output arrow. For operators that require multiple possibilities such as alternation and matching zero, one or more, the generator must split the path to connect the dangling arrows to multiple future states. In alternation, the two preceding expressions are taken and connected in parallel. To match zero or one, the generator must allow for a path through or around the tag. To match one or more, the generator must connect the arrow leading out back into the tag as well as proceeding to the next state. In order to match zero or more, an arrow must be connected 
both around, and looping through the tag. These are generated in Python using state objects

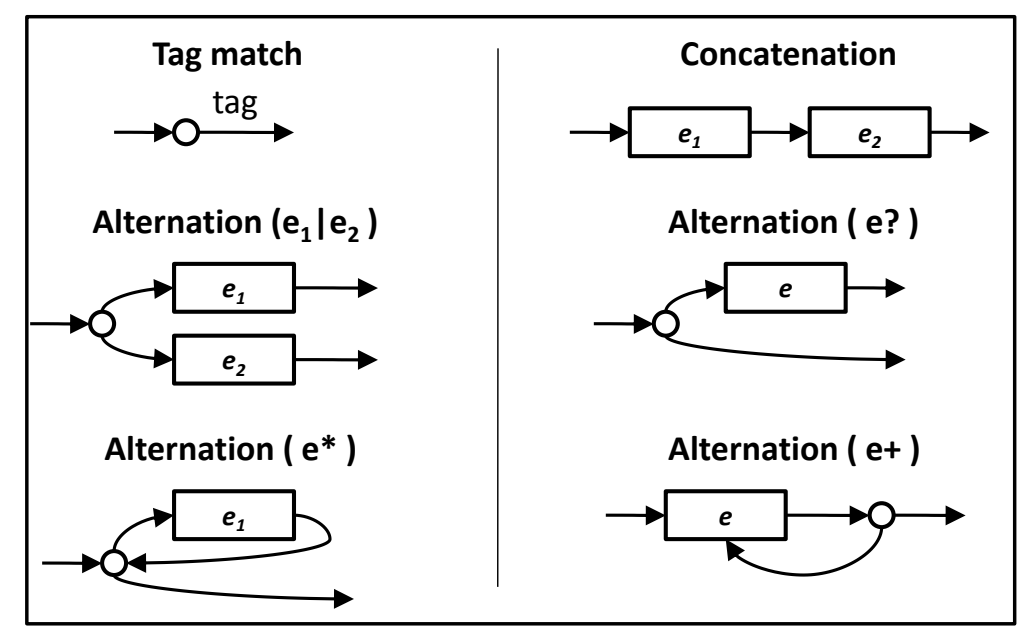

Figure 9 - Non-Finite Automata

with out lists that contain the next states. The out list may contain the state itself in order to simulate arrows that loop back into the state for matching purposes.

In order to emulate the NFA across an array of tokens, we generate 3 lists of states. The first list is the list of current states, which are tested one by one on the current token. The second list is the list of next states, which are added as each state matches. The third list is the list of complete states which are added as terminal matching states are found. The program iterates through each token, and iterates through each current state. As matches are found, they are added to the next-list which is swapped for current list when all current states have been completed. The matching code matches for the part of speech tag, the chunking tag, or the text itself. Using the '^’' concatenator, a match may be across a combination of text, part of 
speech tag, or chunking tag. The negation operator is also handled here by matching across all possibilities and returning false if a match occurs.

When an expression is created to match a clause, the detection may need to continue both after and before the clause itself. This is both useful to limit the number of possible concentric matches and to locate the starting point of a clause when it depends on the tokens that appear before the beginning of the clause. Therefore we have added an open and closed bracket operator. When the NFA reaches one of these operators, the location is noted in the state node and passed along until either a terminal matching node is reached, or the path is dropped from a non-matching state. When a bracket operator is encountered while running the NFA, the NFA then advances to the next state without advancing the token array by adding the out states to the current list for processing. When the completed match list is returned, the start and ending locations are recorded for each matching state.

The expression itself differs from a regular expression in several subtle ways. The smallest unit, which is called a tag, serves the same purpose of a character in a standard regular expression. First, spaces must separate tags not otherwise separated by special characters since each tag in the expression may have multiple characters. Second, the negation operator can only occur on a tag unit and not across multiple tags or a large state set. Third, a tag may be either a part of speech tag name, a full chunking tag including the beginning or internal identifiers, a truncated chunking tag, or a unit of text from the tokenized sentence. The special characters used are a 
pipe (“|”) for alternation, a question mark to match zero or one of the preceding, an asterisk to match zero or more, a plus symbol to match one or more, and a hash or pound symbol (“\#”) for a wildcard. The hash was used for wildcard instead of a period that is the common case because periods are commonly needed for regular expression matching, and no escape character was implemented for this algorithm. The caret symbol (“^^”) is used to match multiple tags on a single token. Parentheses are used to group sets of tags in the expression for infix expression purposes, and square brackets are used to delineate the beginning and ending of the clause in question. When the expression is translated to a postfix style expression, the ampersand symbol ("\&") is used for concatenation.

An example of a simple regular expression to illustrate this is the case of extracting the adjective descriptors from a noun phrase. Consider the sentence, The fat, lasy, orange cat was named Garfield. In this case, we have the noun phrase The fat, lasy, orange cat which is tagged, “The/DT fat/JJ ,/, lazy/JJ ,/, orange/JJ cat/NN". To mark each adjective within a noun phrase starting with a determiner and separated by commas, we would use the expression DT $\left(\mathrm{I}-\mathrm{NP}^{\wedge} \mathrm{JJ},\right)^{*}\left[\mathrm{I}-\mathrm{NP} \mathrm{PJJ}^{\wedge}\right], \mid \mathrm{NN}$. The DT looks for a determiner part of speech tag. The next group handles zero or more instances of an adjective followed by a comma, then the open square bracket marks the beginning of the clause. The I-NP^JJ tag looks for a token that is within a noun phrase and has the part of speech tag for an adjective. This also makes sure that the matching adjective does not appear in a separate noun phrase. The closing square bracket marks the end of the clause, and the , INN matches a comma or noun part of speech tag after 
the adjective. If there is no comma or noun after the adjective, then the expression will not match.

\subsection{S E N T E N C E TRA N S F R M A T I O N S}

Sentence transformations take place in a multi-step process that identifies the clause location and type, the connecting phrase, and main verb for transformation if necessary. The identification process uses the regular expression engine to identify each type. The clause types handled in this system are restrictive and non-restrictive relative clauses, appositives, and sequences. Regular expressions identifying each were created using Siddharthan's algorithms (Siddharthan 2003), simple English grammar tests from various websites, and CoNLL 2001 clausal boundary data (Dejean and Sang 2001). The regular expressions were formed as an initial step in order to allow for machine learning techniques to improve on their accuracy.

\subsubsection{RELATIVE CLAUSES}

Relative clauses are clauses which begin with a relative pronoun and give additional information about a noun in the sentence. An example of a relative clause is The man, who had red hair, was the owner of the store. In this case, we wish to identify the relative clause who had red

hair, and the attached noun The man, to form the sentences The man had red hair and The man was 
the owner of the store. In this system we use the following regular expression to identify relative clauses:

$$
\text { , [RC CC?RL(, \#+, )?(((NP|PP)+, }
$$

$\left(N R^{\wedge} N P \mid N R^{\wedge} P P\right)\left(N R^{\wedge} N P|P P| A D J P \mid V B G\right) *\left(R L(V P|N P| P P|A D J P| A D V P)\left(N R^{\wedge} N P|P P| A D J P\right.\right.$

$$
\text { |VBG } \left.\left.\mid A D V P)^{*}\right)^{*}\right)|(R B|R B R| R B S, R B|R B R| R B)|(J J|J J R| J J S, J J|J J R| J J S) \mid(\text {, (B-VP^VBN|B- }
$$

\section{$\left.\left.\left.\left.\mathrm{VP}^{\wedge} \mathrm{VBG}\right)\left(\mathrm{I-V} \wedge \mathrm{P}^{\wedge} \mathrm{BN} \mid \mathrm{I-V} \mathrm{P}^{\wedge} \mathrm{VBG}\right) * ! \mid-\mathrm{VP}\right) \mid ! \mathrm{O}^{*}\right)^{*} \mathrm{RC}\right](, \mid$.}

The expression begins with a comma to begin the relative clause and then we mark the beginning of the clause. The CC marks "and" or "in" for expressions like "and who..." before matching for the relative pronoun. In this case, relative pronouns are "who", "whom", "whose", and "which". The relative pronoun "that" is not considered because of its ambiguity. In the case of an aside, a comma delineated phrase is allowed directly after the relative pronoun. We then expect a noun phrases with constituent prepositional phrases. This is multiple due to the nature of chunking. We then allow for several types of phrases, including internal relative clauses. Lastly, strings of adverbs and adjectives with internal commas are allowed before closing the relative clause at a comma or a period. This was patterned after the algorithm outlined by Siddharthan, which performed at an accuracy of $91 \%$, the best CoNLL task on relative clause identification by comparison was $81 \%$ (Marquez 2003). The regular expression performed similarly. 


\subsubsection{APPOSITIVES}

Appositives are nouns that explain or identify nearby nouns. An example of a sentence with an appositive is, My brother's car, [Appos a sporty convertible with bucket seats Appos], is the enyy of my friends. Since an appositive is equivalent to the noun it is explaining or identifying, we can create several alternative sentences: My brother's car is the enyy of my friends. , A sporty convertible with bucket seats is the envy of my friends., and My brother's car is a sporty convertible with bucket seats. We use the following regular expression in this system to identify appositives:

$$
(\mathrm{NP} \mid \mathrm{PP})+
$$

$\left[A p p o s\left(N R^{\wedge} N P^{\wedge} ! S T\left|N R^{\wedge} P P^{\wedge} ! I N\right| V B G|V B N| A D J P\right)\left(N R^{\wedge} N P|P P| A D J P|V B G| V B N \mid(\right.\right.$,

$$
\left.\mathrm{ST}))^{*}\left(\mathrm{RL}(\mathrm{VP}|\mathrm{NP}| \mathrm{PP}|\mathrm{ADJP}| \mathrm{ADVP})(\mathrm{NR} \wedge \mathrm{NP}|\mathrm{PP}| \mathrm{ADJP}|\mathrm{VBG}| \mathrm{ADVP})^{*}\right)^{*} \mathrm{Appos}\right]((, \mathrm{IST}) \mid .)
$$

First, the appositive must come after a noun phrase and its constituent prepositional phrases. We begin the appositive after a comma, but the appositive can begin with a noun phrase as long as it's not a relative pronoun or state name, a prepositional phrase as long as it's not a subordinating conjunction or relative pronoun, a verb in present or past participle form, or an adjectival phrase. State logic was added in to prevent false positives in "city, state" form. This probably should be expanded to all location names for comprehensive coverage. We then allow for a continuation of the noun phrase with prepositional, or adjectival phrases, and internal relative clauses. Verbs in base or present form will stop the system from identifying a relative clause. Finally, we end the appositive with a comma or period, making sure that we are not ending with a state name. 


\subsubsection{SEQUENCES}

Sequences are three or more phrases which refer to the same object within a sentence. An example of a sequence is: The man on the pier was wearing sunglasses, a fisherman's cap, a bawaiian shirt, and flip-flops. We can create several sentences based on the number of elements in the sequence: The man on the pier was wearing sunglasses., The man on the pier was wearing a fisherman's cap., etc. This system can identify more complex sequences such as: It is proposing [Seq increased tax rebates for textiles and labor-intensive products Seq], [Seq expanded bank lending to small businesses Seq], [Seq reduced taxes on housing transactions Seq], and [Seq speeded-up infrastructure construction Seq]. The identification of sequences are three separate regular expressions: one for nouns, one for pronouns, and one for verbs and adjectives. This is to match the beginning of each sequence

item, and to handle the differences in complexity between each type of sequence. For instance, in order to match the verb and adjective sequence, we use the regular expression:

VP ([Seq(VBN|JJ) (NR^NP|NR^PP|VBG |VBN|ADJP) (NR^NP|PP|ADJP|VBG |VBN|CC|(, $S T))^{*}(R L(V P|N P| P P|A D J P| A D V P)(N R \wedge N P|P P| A D J P|V B G| A D V P) *)^{*}$ Seq] ,)+ ([Seq(VBN|JJ) (NR^NP|NR^PP|VBG |VBN|ADJP) (NR^NP|PP $|A D J P| V B G|V B N| C C \mid$, $\left.\mathrm{ST}))^{*}\left(\mathrm{RL}(\mathrm{VP}|\mathrm{NP}| \mathrm{PP}|\mathrm{ADJP}| \mathrm{ADVP})\left(\mathrm{NR} \mathrm{R}^{\wedge} \mathrm{NP}|\mathrm{PP}| \mathrm{ADJP}|\mathrm{VBG}| \mathrm{ADVP}\right)^{*}\right)^{*} \mathrm{Seq}\right]$, ?)? CC $[$ Seq(VBN|JJ) (NR^NP|NR^PP|VBG $|V B N| A D J P)\left(N R^{\wedge} N P|P P| A D J P|V B G| V B N|C C|\right.$, $S T))^{*}\left(R L(V P|N P| P P|A D J P| A D V P)\left(N^{\wedge} N P|P P| A D J P|V B G| A D V P\right)^{*}\right)^{*}$ Seq] $(V P \wedge ! V B N \mid$. 
The length of the expression is to make sure to capture the beginning, middle, and ending states of the sequence. In this expression, we begin with a verb phrase and match either the past participle verb or adjective and then match a sequence similar to an appositive. The difference between each state is the placement of commas or a coordinating conjunction such as "and" or "or". To end this type of sequence, we match on a verb phrase that does not begin with the past participle form, or with the end of the sentence.

\subsubsection{U N M R K E D C L A S E S}

When there are no commas surrounding a clause, it is more difficult to identify in a sentence. The sentence, The car sliding out of control toward the building will likely bit the window, contains a participial phrase that can be treated similarly to appositives. The participial phrase, sliding out of control toward the building, may be removed from the sentence without affecting the meaning or correctness of the sentence. It also may be placed equal to the attached noun phrase. In identifying participial phrases, the algorithm first finds a noun phrase followed by a verb in gerund form. Further noun phrases, relative clauses, and verbs in gerund form are allowed, ending the clause with a verb phrase or end of sentence.

Another common unmarked clause is an unmarked appositive. An appositive may have commas surrounding it, or may be placed in the sentence unmarked with the assumption that the native speaker will understand its context. Consider the sentence: Evan's friend John cheated on the test. The word John is an appositive, and the sentence could be rendered: Evan's friend, 
John, cheated on the test. This sequence can be identified without commas by finding a proper set of NP-NP sequences in a row. The regular expression that identifies both unmarked appositives and unmarked participial phrases is:

((B-NP I-NP* B-NP^POS I-NP+)|(B-NP^!POS I-NP* I-NP^!DT)|B-NP^!DT^NR^!RB) [Appos $\left(N R^{\wedge} B-N P^{\wedge} D T\left|N R^{\wedge} B-N P^{\wedge} N N P\right| N R^{\wedge} B-N P^{\wedge} N N P S\left|\left(V B G \mid-V P^{*}(N P \mid P P)\right)\right| V B N \mid-V P^{*}\right.$ $N P)(N R \wedge N P|P P| A D J P|V B G|(T O \mid-$ $\left.\mathrm{VP}+)^{*}\right)^{*}\left(\mathrm{RL}(\mathrm{VP}|\mathrm{NP}| \mathrm{PP}|\mathrm{ADJP}| \mathrm{ADVP})\left(\mathrm{NR} \mathrm{R}^{\wedge} \mathrm{NP}|\mathrm{PP}| \mathrm{ADJP}|\mathrm{VBG}| \mathrm{ADVP}\right)^{*}\right)^{*} \mathrm{Appos}$ (B-VP|.)

The first part makes sure to combine noun phrases that are connected with a possessive. Technically, in our example Evan and 's friend are two separate noun phrases and may be treated as separate entities based on phrase structure. For the purposes of splitting clauses into sentences, we want to attach Evan's friend to John as one unit.

Restrictive relative clauses are similar to unmarked appositives in that they are not surrounded by commas. We can detect restrictive relative clauses by looking for the relative pronoun marking the beginning of the clause. A sentence like I told you about the woman who lives next door. is separated into the sentences I told you about the woman. The woman lives next door. The following is able to find restrictive relative clauses.

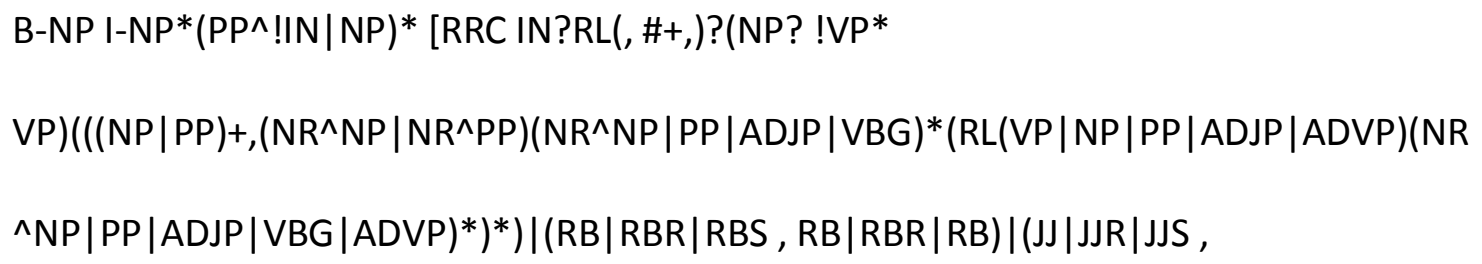




\section{$\mathrm{JJ}|J J R| J J S) \mid\left(,\left(B-V P^{\wedge} V B N \mid B-V P^{\wedge} V B G\right)\left(I-V P^{\wedge} V B N \mid I-V P^{\wedge} V B G\right)^{*} ! \mid-\right.$ VP) $\left.\left.\mid\left(N^{\wedge} N P|A D J P| A D V P \mid S B A R\right)^{*}\right)^{*} R R C\right](,|:| ;|| B-.V P \mid R L)$}

We can also detect unmarked contact clauses like in the sentence: The book we ordered was very expensive. This allows us to generate the sentences: The book was very expensive. We ordered the book. Since a contact clause is a type of relative clause, we use a modified relative clause regular expression to find it.

(B-NP I-NP B-VP I-VP*!PP*|B-VP I-VP*!PP*)? [Attach B-NP I-NP* Attach] [Contact SBAR? $\left(B-N P^{\wedge} ! P O S I-N P^{*}\right)\left(\left(P P^{*} N P+\right) ? ! V P * V P+N P *(P P+N P *)^{*}\right)$ Contact](.|B-VP|RL)

The Attach tag is used to identify where the attached noun phrase is located.

Unfortunately, unmarked appositives, restrictive relative clauses, and contact clauses were not found in large enough quantities in the test data to give a valid measurement. The system was able to handle a sampling found on the internet located in appendix 1. 


\section{SYSTEM EVALUATION}

Evaluation of an information retrieval algorithm is usually done through three different measures: precision, recall, and F-measure (Rijsbergen 1979). Precision and recall are both represented as percentages while F-measure is represented as a single number. Precision is the number correct over the total marked. Recall is the number correct over the total number of instances in the data. F-measure is the harmonic mean of precision and recall: $F_{\beta}=2 \frac{\beta^{2} P R}{\beta^{2} P+R}$. This is usually computed with $\beta=1$. Changing the value of $\beta$ weights the Fmeasure score toward either precision or recall. A lower $\beta$ favors precision while a larger $\beta$ favors recall. TREC 2003 QA track used $\beta=5$ to give recall 5 times the weight of precision in its definition question section.

In order to evaluate the system, the first 1,000 sentences of CoNLL 2001 test data were analyzed by hand to identify whether each of the three clausal types were identified correctly, missed, or whether there was a clause incorrectly identified. The results in Figure 10 show

\begin{tabular}{|l|l|}
\hline Algorithm & RC Recall \\
\hline Top Scoring CoNLL 200I shared task (perceptrons) & $81 \%$ \\
\hline Siddharthan (hand coded) & $91 \%$ \\
\hline Miller (regular expression) & $90 \%$ \\
\hline
\end{tabular}

Figure 10 - Relative Clause Identification Comparison 
that the relative clause results roughly match the results of Siddharthan, which is similar to the best recent results (Van Nguyen, Nguyen and Shimazu 2007), showing a similarly high recall. The F-measure was calculated with a $\beta$ of 1 . Overmarks were most common on sequences with titles such as "Mr. Smith, President and CEO of...". Changes in writing style also were problematic. In the CoNLL data, there are both news articles and instructions. While news articles were generally parsed correctly, the instruction sections were not. Other sources of error were references to time and locations not covered by day and state identifiers, as well as stock market specific language such as, "B.A.T ended the day at 778, [Appos down 5 Appos], on turnover of 7.5 million shares." These presumably could be improved through more external reference information.

Transformations conducted on the same data showed only a handful of errors when clauses were identified correctly, mostly due to the simple structure of the majority of the sentences in the source data. The attachment algorithm is a basic nearest phrase picker, so complex sentence structure will cause the transformation to fail, but this occurred rarely.

\begin{tabular}{|l|r|r|r|r|r|r}
\hline Identification & Correct & Miss & Overmark & Recall & Precision & F-measure \\
\hline Appos & 99 & 13 & 35 & $88.39 \%$ & $73.88 \%$ & 0.80 \\
\hline Seq & 19 & 8 & 9 & $70.37 \%$ & $67.86 \%$ & 0.69 \\
\hline RC & 27 & 3 & 5 & $90.00 \%$ & $84.38 \%$ & 0.87 \\
\hline
\end{tabular}

Figure 11 - Clause Identification Results 
Other clauses were attempted as well as the three listed above, but did not occur in the test corpus, since newspaper accounts tend to have a narrow grammar. A sentence such as "The photographer could not develop the pictures I had taken in Australia," can be broken down by the system into: "The photographer could not develop the pictures," and "I had taken the pictures in Australia." This transformation turned out to be less common than expected and was dropped from the results when no matching sentences were found in the test data. 


\section{FUTURE WORK}

The system currently has several limitations such as inability to distinguish references to time and location and inability to handle complex attachment. The ability to reliably identify clauses within a single regular expression means that it should be possible to further advance the algorithm through machine learning techniques such as TBL (Li, et al. 2008). Furthermore, the system may be able to identify other sentence features reliably for other purposes than clausal extraction, such as changing a sentence to and from passive voice, moving NP's and other grammatical transformations useful for paraphrasing or making sentence structure uniform across multiple sources.

A future project may be to apply a TBL or other machine learning algorithm to generate regular expression strings identifying specific clauses more reliably. Another may be to incorporate this system into a search engine or question answering system and compare the accuracy of the resulting data. A final project would be to expand the possible clause types and test their results on different styles of writing, such as forum posts and encyclopedia articles. 


\section{CONCLUSIONS}

This system serves as an initial step for text to text tasks such as summarization, translation, and paraphrase engines. Data on the web tends to be in complex natural language form such as "Sao Paulo, the world's second-largest city, has a population of just over ten million. Three other cities, Bombay, Jakarta and Karachi, have grown to more than nine million people." Extracting clauses allows for systems which focus on accuracy to be more precise and allows for an extra dimension in systems that rely primarily on keywords. We have demonstrated a system with high accuracy that is able to both identify and extract information from complex sentences relying on the grammatical structure. Furthermore, we have moved in the direction from rule-based algorithms to machine learning on complex structures proving that it is possible to match hand-coded algorithms with a type of regular expression string.

While certain semantic information would be useful to add and would cut down sharply on

error rates, the results based on the tests conducted are better than all but hand-coded methods, bringing complex grammatical analysis near to practical use. 


\section{BIBLIOGRAPHY}

Bird, Edward Loper and Steven. "NLTK: The Natural Language Toolkit." In Proceedings of the ACL Workshop on Effective Tools and Methodologies for Teaching Natural Language Processing and Computational Linguistics. Philadelphia: Association for Computational Linguistics, 2002.

Brill, Eric. "A simple rule-based part-of-speech tagger." 3rd Conference on Applied Natural Language Processing. 1992. 152 - 155.

Brill, Eric. "Learning to Parse with Transformations." In Recent Advances in Parsing Technology, by Harry C. Bunt and Masaru Tomita, 221 - 240. Springer, 1996.

Brill, Eric. "Recent Advances in Parsing Technology." In Learning to Parse with Transformations, by Marsaru Tomita Harry Bunt. Dordrecht: Kluwer Academic Publishers, 1996.

Brill, Eric. "Transformation-based error-driven learning and natural language processing: A case study in part of speech tagging." Computational Linguistics, 1995: 543-565.

Carroll, John, Guido Minnen, Darren Pearce, Yvonne Canning, Siobhan Devlin, and John Tait. "Simplifying text for language-impaired readers." 9th Conference of the European Chapter of the ACL. Bergen, Norway, 1999.

Chandrasekar, Raman, Christine Doran, and Bangalore Srinivas. "Motivations and Methods for Text Simplification." COLING. 1996. 1041-1044.

Chomsky, Noam. "Three models for the description of language." Information Theory, IEEE Transactions on 2, no. 3 (1956): 113-124.

Dejean, Herve, and Erik F. Tjong Kim Sang. "Introduction to the CoNLL-2001 Shared Task: Clause Identification." Edited by Walter Daelemans and Remi Zajac. Proceedings of CoNLL2001. Toulouse, France, 2001. 53-57.

Earley, Jay. "An Efficient Context-Free Parsing Algorithm." PhD Thesis, Carnegie-Mellon University, 1968.

Erkan, Gunes, and Dragomir R. Radev. "LexRank: Graph-based Lexical Centrality as Salience in Text Summarization." Journal of Artificial Intelligence Research, 2004: 457-479.

Florian, Radu, and Grace Ngai. "Transformation-based learning in the fast lane." North American Chapter of the Association for Computational Linguistics. Pittsburgh, 2001. 40-47.

Francis, W. Nelson, and Henry Kucera. Frequency Analysis of English Usage: Lexicon and Grammar. Boston: Houghton Mifflin, 1982.

Jinxi Xu, Ralph Weischedel , Ana Licuanan. "Evaluation of an extraction-based approach to answering definitional questions." 27th annual international ACM SIGIR conference on Research and development in information retrieval. Sheffield, United Kingdom, 2004. 418 - 424.

Kavallieratou, Ergina, Efstathios Stamatatos, Nikos Fakotakis, and George Kokkinakis. "Handwritten Character Segmentation using Transformation-based Learning." International Conference on Pattern Recognition. Barcelona, 2000. 634-637. 
Klebanov, B. Beigman, Kevin Knight, and Daniel Marcu. "Text Simplification for Information-Seeking Applications. On the Move to Meaningful Internet Systems." Lecture Notes in Computer Science 3290, 2004: 735-747.

Knight, Kevin, and Daniel Marcu. "Statistics-Based Summarization - Step One: Sentence Compression." Seventeenth National Conference on Artificial intelligence and Twelfth Conference on innovative Applications of Artificial intelligence. AAAI Press / The MIT Press, 2000. 703-710.

Li, Yunyao, Rajasekar Krishnamurthy, Sriram Raghavan, and Shivakumar Vaithyanathan. "Regular Expression Learning for Information Extraction." Proceedings of the 2008 Conference on Emperical Methods in Natural Language Processing. Honolulu, 2008. 21-30.

Liu, Hugo. MontyTagger. 2003. http://web.media.mit.edu/ hugo/montytagger.

Mani, Inderjeet, and Mark T. Maybury. Advances in Automatic Text Summarization. Cambridge, MA: MIT Press, 1999.

Marcu, Daniel and Soricut, Radu. "Towards Developing Generation Algorithms for Text-toText Applications." Association for Computational Linguistics Conference. Ann Arbor, MI, 2005.

Mark-Jan Nederhof, Giorgio Satta. "IDL-Expressions: A Formalism for Representing and Parsing Finite Languages in Natural Language Processing." J. Artif. Intell. Res. (JAIR), 2004: 287-317.

Marquez, Xavier Carreras and Lluis. "Phrase Recognition by Filtering and Ranking with Perceptrons." Borovets, Bulgaria: Proceedings of the International Conference on Recent Advances in Natural Language Processing, 2003.

Martin, Daniel Jurafsky and James H. Speech and Language Processing: An Introduction to Natural Language Processing, Computational Linguistics and Speech Recognition. Upper Saddle River, N.J.: Prentice Hall, 2000.

Ouhalla, Jamal. Introducing Transformational Grammar - From Principles and Parameters to Minimalism. New York, NY: Oxford University Press, Inc., 1999.

Palmer, Marc Vilain and David. "Transformation-based bracketing: Fast algorithms and experimental results." Workshop on Robust Parsing, held at ESSLLI. 1996. 93 - 102.

Ramshaw, Lance A., and Mitchell P. Marcus. "Exploring the statistical derivation of transformational rule sequences for part-of-speech tagging." Balancing Act Workshop on Combining Symbolic and Statistical Approaches to Language. Association for Computational Linguistics, 1994. 86-95.

—. "Text Chunking using Transformation-Based Learning." ACL Third Workshop on Very Large Corpa. 1995. 82-94.

Rijsbergen, Cornelis J. Van. Information Retrieval 2ed. London: Butterworth's, 1979.

Sang, Erik F. Tjong Kim, and Sabine Buchholz. "Introduction to the CoNLL-2000 shared task: Chunking." Conference on Computational Natural Language Learning. 2000. 127-132.

Santorini, Beatrice. Part-of-Speech Tagging Guidelines for the Penn Treebank Project. Department of Computer and Information Science, University of Pennsylvania, 1991.

Siddharthan, Advaith. "Syntactic Simplification and Text Cohesion." PhD thesis, University of Cambridge, UK., 2003.

Thompson, Ken. "Programming Techniques: Regular expression search algorithm." Communications of the ACM, Vol 11, 1968: 419-422. 
Van Nguyen, Vinh, Minh Le Nguyen, and Akira Shimazu. "Using Conditional Random Fields for Clause Splitting." Proceedings of The Pacific Association for Computational Linguistics. Melmourne, Australia, 2007. 58-65.

Voorhees, Ellen. "Overview of the TREC 2003 Question Answering track." TREC 2003. 2004.

—. "Overview of the TREC 2004 Question Answering track." TREC 2004. 2005.

-. "The TREC-8 Question-Answering Track Report." In Proceedings of TREC-8. Gaithersburg, MD: National Institute of Standards and Technology, 1999. 77-82. 
Examples are from grammar tests and other sentences found in various locations on the internet. Only output is shown with identification first and extracted sentences following. Input was unmarked sentences. The system adds bracketed markings with keywords for identification. Extracted sentences are on the following lines with each algorithm labeled. Some minor errors include extraneous commas, and incorrect capitalization. Other errors are noted with each example.

[Attach Her husband Attach], [Appos Fritz Appos], is a nice guy . Added Appos sentence: Her husband is Fritz. Added Appos sentence: Her husband, is a nice guy.

The firm chose [Attach Mary Attach], [Appos vice president of public affairs Appos], as its chief executive officer. Added Appos sentence: Mary is vice president of public affairs . Added Appos sentence: The firm chose Mary, as its chief executive officer.

[Attach The Grand Canyon Attach], [Appos one of our nation 's most popular tourist attractions Appos], is breathtaking to behold. Added Appos sentence: The Grand Canyon is one of our nation 's most popular tourist attractions.

Added Appos sentence: The Grand Canyon, is breathtaking to behold.

[Attach Nej1 Armstrong Attach], [Appos the first man who walked on the moon Appos], is a native of Ohio.

Added Appos sentence: Nei1 Armstrong is the first man who walked on the moon .

Added Appos sentence: Nei1 Armstrong, is a native of ohio .

[Attach Evan 's friend Attach] [Appos2 John Appos2] cheated on the test

Added Appos2 sentence: Evan 's friend is John.

Added Appos2 sentence: Evan 's friend cheated on the test.

[Attach The car Attach] [Appos2 sliding out of control toward the building Appos2] wi11 likely hit the window .

Added Appos2 sentence: The car is sliding out of control toward the building.

Added Appos2 sentence: The car wi11 likely hit the window . 
Cameron spotted [Attach his brother Attach] [Appos2 throwing rocks at the passing cars Appos2].

Added Appos2 sentence: his brother is throwing rocks at the passing cars.

Added Appos2 sentence: Cameron spotted his brother.

[Attach The astronaut Attach] [Appos2 chosen to ride the space shuttle to Mars Appos2] is afraid of heights .

Added Appos2 sentence: The astronaut is chosen to ride the space

shutt le to Mars.

Added Appos2 sentence: The astronaut is afraid of heights .

[Attach2 The book Attach2] [RRC2 we ordered RRC2] was very expensive . Added RRC2 sentence: we ordered The book.

Added RRC2 sentence: The book was very expensive.

The paint on [Attach2 the bench Attach2] [RRC2 you are sitting on RRC2] is sti11 wet.

Added RRC2 sentence: you are sitting on the bench.

Added RRC2 sentence: The paint on the bench is stil1 wet.

(In the following example, the first sentence needs the attached noun phrase to occur within the relative clause instead of after to read more fluid7y.)

The photographer could not develop [Attach2 the pictures Attach2] [RRC2 I had taken in Australia RRC2].

Added RRC2 sentence: I had taken in Australia the pictures.

Added RRC2 sentence: The photographer could not develop the pictures. It is proposing [Seq increased tax rebates for textiles and labor-

intensive products Seq], [Seq expanded bank lending to smal1 businesses

Seq], [Seq reduced taxes on housing transactions Seq], and [Seq

speeded-up infrastructure construction seq].

Added Seq sentence: It is proposing increased tax rebates for textiles and labor-intensive products.

Added Seq sentence: It is proposing expanded bank lending to smal1

businesses.

Added Seq sentence: It is proposing reduced taxes on housing

transactions.

Added Seq sentence: It is proposing speeded-up infrastructure construction .

(The first added sentence does not factor in the plural or the 7ead in "such as")

Ship companies carrying [Attach bulk commodities Attach], [Appos such as [Seq oi $1 \mathrm{Seq}$, [Seq grain Seq], [Seq coal Seq] and [Seq iron ore Appos] Seq], have been able to increase their rates in the last couple of years.

Added Appos sentence: bulk commodities is such as oi1, grain, coal and iron ore.

Added seq sentence: ship companies carrying bu1k commodities, such as oil, have been able to increase their rates in the last couple of years 
Added Seq sentence: Ship companies carrying bulk commodities, such as grain, have been able to increase their rates in the last couple of years.

Added Seq sentence: Ship companies carrying bulk commodities, such as coal, have been able to increase their rates in the last couple of years.

Added Seq sentence: Ship companies carrying bulk commodities, such as iron ore, have been able to increase their rates in the last couple of years.

Added Appos sentence: Ship companies carrying bulk commodities, have been able to increase their rates in the last couple of years.

Share prices closed higher in [Seq Sydney Seq], [Seq Taipei Seq], [Seq Wel1ington Seq], [Seq Manila Seq], [Seq Hong Kong Seq] and [Seq Singapore Seq] and were lower in seoul.

Added Seq sentence: Share prices closed higher in sydney and were lower in Seoul.

Added Seq sentence: Share prices closed higher in Taipei and were lower in Seou1.

Added Seq sentence: Share prices closed higher in wellington and were Tower in seoul.

Added Seq sentence: Share prices closed higher in Manila and were lower in Seou1.

Added Seq sentence: Share prices closed higher in Hong Kong and were lower in Seoul.

Added Seq sentence: Share prices closed higher in singapore and were lower in Seoul.

[Attach Sao Paulo Attach], [Appos the world 's second-1argest city Appos], has a population of just over ten mil1ion.

Added Appos sentence: Sao Paulo is the world 's second-largest city . Added Appos sentence: Sao Paulo, has a population of just over ten mi17ion.

(The complexity of forming new sentences causes severa 7 grammatica 7 errors here after successfuly identifying the clauses)

[Attach Three other cities Attach], [Appos [Seq Bombay Seq], [Seq Jakarta Seq] and [Seq Karachi Appos] Seq], have grown to more than nine mil1ion people.

Added Appos sentence: Three other cities is Bombay, Jakarta and Karachi .

Added Seq sentence: Three other cities, Bombay, have grown to more than nine milition people.

Added Seq sentence: Three other cities, Jakarta, have grown to more than nine million people.

Added Seq sentence: Three other cities, Karachi, have grown to more than nine million people.

Added Appos sentence: Three other cities, have grown to more than nine mi11ion people.

The company already markets a wide range of [Seq detergents Seq], [Seq food Seq], [Seq household Seq] and [Seq health-care products Seq] . Added Seq sentence: The company already markets a wide range of detergents.

Added seq sentence: The company already markets a wide range of food. 
Added seg sentence: The company already markets a wide range of household.

Added seq sentence: The company already markets a wide range of healthcare products.

Simpler English Wikipedia:

This is the first section of the Oklahoma article of the simple English Wikipedia as processed through the system:

ok1ahoma is [Attach2 a state Attach2] that [RRC2 is in the southern part of the Central United States RRC2].

Added RRC2 sentence: is in the southern part of the Central United

States a state.

Added RRC2 sentence: Ok1ahoma is a state.

It had a population of about $3,617,000$ people in 2007 .

The state has a 1and area of about $68,667 \mathrm{sq} \mathrm{mi}$.

ok1ahoma is the 28th largest state by population.

It is the 20th largest state by area.

The name of the state comes from the choctaw words okla and humma.

It means 'Red People ''.

It is also known by its nickname, The sooner state.

(The number "1907" was mistagged as a noun rather than a cardinal number, which threw off the system)

The state was formed from [Attach Indian Territory on [Attach November 16 Attach] Attach] , [Appos [Appos 1907 Appos] Appos]

Added Appos sentence: Indian Territory on November 16 is 1907 .

Added Appos sentence: November 16 is 1907 .

Added Appos sentence: The state was formed from Indian Territory on

November 16 .

Added Appos sentence: The state was formed from Indian Territory on November 16 .

It was the 46th state to become part of the United States.

[Attach2 The peop1e Attach2] who [RRC2 1ive in the state RRC2] are known as Ok1ahomans.

Added RRC2 sentence: Tive in the state The people.

Added RRC2 sentence: The people are known as oklahomans.

The state 's capital and largest city is oklahoma City.

oklahoma is a large producer of [Seq natural gas Seq], [Seq oil seq]

and [Seq food Seq].

Added Seq sentence: Ok1ahoma is a large producer of natural gas.

Added Seq sentence: ok 1 ahoma is a large producer of oi 1 .

Added Seq sentence: Oklahoma is a large producer of food.

(The first element of the sequence is misidentified, throwing off the 7ater sentences)

It has [Seq large industries in aviation seq], [Seq energy seq], [Seq telecommunications Seq], and [Seq biotechnology Seq].

Added Seq sentence: It has large industries in aviation . Added Seq sentence: It has energy. 
Added Seq sentence: It has telecommunications .

Added seq sentence: It has biotechnology.

The state has one of the fastest growing economies in the nation .

Between 2005 and 2006 , it had the third highest percentage of income

growth and the highest percentage in gross domestic product growth .

Ok1ahoma city and Tulsa are the main economic areas of ok lahoma.

Almost 60 percent of oklahomans live in these two metropolitan

statistical areas.

Oklahoma has [Seq smal1 mountain ranges Seq], [Seq prairies Seq], and [Seq eastern forests Seq].

Added Seq sentence: Oklahoma has smal1 mountain ranges.

Added Seq sentence: Oklahoma has prairies.

Added Seq sentence: Oklahoma has eastern forests.

Most of Oklahoma is in the Great Plains.

It is regularly hit by severe weather.

(Grammatica77y ambiguous wording in the following sentence cause a misidentification of the first sequence element, and follows to incorrect derived sentences)

The cultural heritage of ok1ahoma is [Seq affected by a population descending from German Seq], [Seq Irish, British Seq] and [Seq Native American people Seq].

Added Seq sentence: The cultural heritage of oklahoma is affected by a population descending from German .

Added Seq sentence: The cultural heritage of oklahoma is Irish, British.

Added Seq sentence: The cultural heritage of oklahoma is Native American people.

More than 25 Native American languages are spoken in oklahoma.

This is more than in any other state.

(The unrecognized sequence causes confusion in the system for the appositive identification algorithm)

In the past, the state was used as [Attach a path for [Attach cattle drives Attach] Attach], [Appos [Appos a place for southern settlers Appos] Appos], and a government-made territory for Native Americans. Added Appos sentence: a path for cattle drives is a place for southern settlers.

Added Appos sentence: cattle drives is a place for southern settlers. Added Appos sentence: In the past, the state was used as a path for cattle drives, and a government-made territory for Native Americans. Added Appos sentence: In the past, the state was used as a path for cattle drives, and a government-made territory for Native Americans. oklahoma is part of the Bible Belt.

Many people believe in evangelical Christianity .

oklahoma is one of the most politically conservative states, but voter registration is largest for the Democratic Party. 